\title{
层子集团折垔位和 $\boldsymbol{n}-\boldsymbol{p}$ 系统
}

郑玉明 和 音 卢兆启

(中国科学院原子能研究所)

核子是由层子组成的, 我们把核子看作层子集团;层子之间存在着很强的相互作用, 我们 假定核力是由集团间的折叠位 ${ }^{[3]}$ 所给出. 本文以此观点来探索核力与层子相互作用之间的联 系,并对 $n-p$ 系统进行具体计算.

定义两层子集团间的相互作用位为 $V_{F}(\boldsymbol{R})$

$$
\begin{aligned}
& V_{F}(\boldsymbol{R})=\left\langle\Psi_{A}|\hat{V}| \Psi_{A}\right\rangle+U(\boldsymbol{R}), \\
& U(\boldsymbol{R})=\left\langle\Psi_{A}\left|\sum_{i=1}^{2} \hat{V}_{A_{i}}\right| \Psi_{A}\right\rangle_{\boldsymbol{R}}-\left\langle\Psi_{A}\left|\sum_{i=1}^{2} \hat{V}_{A_{i}}\right| \Psi_{A}\right\rangle_{\infty}, \\
& \boldsymbol{R}=\left\langle\Psi_{A}\left|\boldsymbol{R}_{12}\right| \Psi_{A}\right\rangle, \\
& \Psi_{A}=N_{A}^{\prime} \mathscr{A}^{\prime}\left(\Psi_{A_{1}} \cdot \Psi_{A_{2}}\right),
\end{aligned}
$$

其中, $\Psi_{A_{i}}$ 是描写由 $A_{i}$ 个层子组成的第 $i$ 个孤立层子集团的反对称波函数,它是由 $S U_{3}^{(\mathrm{c})} \times S U_{2}^{(\tau)}$ $\times S U_{2}^{(\sigma)}$ 色、味、自旋函数及 $(1 S)^{3}$ 谐振子波函数构成的 ${ }^{[2]}$. 对于核子 $p 、 n, A_{i} \equiv 3$, 而且只由 $u 、 d$ 两味层子组成,故单核子波函数 $\Psi_{A_{i}}$ 可写为

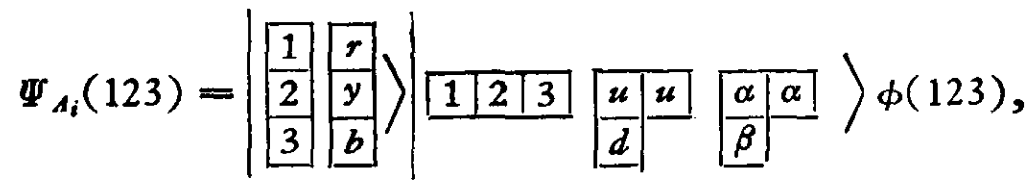

$$
\begin{aligned}
& \phi(123)=\left(\frac{1}{\sqrt{\pi} b_{0}}\right)^{3 / 2} \cdot \exp \left[-\frac{1}{2 b_{0}^{2}} \sum_{j=1}^{3}\left(r_{i}-\frac{z}{2}\right)^{2}\right],
\end{aligned}
$$

这里, $r 、 y 、 b$ 表示红、黄、蓝三种颜色, $\alpha 、 \beta$ 表示自旋向上、下, 空间波函数 $\phi(123)$ 取为双阱 集团模型波函数 ${ }^{[3]}, z / 2$ 是阱心坐标, $z$ 为两阱心之间的距离, $b_{0}$ 为势阱参数. $\Psi_{A}$ 是描写组成 两层子集团的系统 $\left(A=A_{1}+A_{2}\right)$ 的反对称波函数, $\mathscr{A}^{\prime}$ 是两团间的反对称化算符, $N^{\prime}$ 是归 一化因子. $\boldsymbol{R}_{12}=\boldsymbol{R}_{1}-\boldsymbol{R}_{2}$, 是两团质心间的距离. $\hat{v}=\sum_{\mu=1}^{A_{1}} \sum_{\nu=A_{1}+1}^{A} \hat{v}_{\mu \nu}$, 是两层子集团之间: 的层子一层子相互作用算符. $\hat{V}_{A_{i}}=\frac{1}{2} \sum_{\nu \neq \mu=1}^{A_{i}} \hat{V}_{\mu \nu}$, 是第 $i$ 团的内部位能算符. $U(\boldsymbol{R})$ 是两孤 立层子集团由于另一团的存在而引起的内部层子相互作用势能的变化之和.

层子之间的相互作用 $\hat{V}_{\mu \nu}$ 取形式

$$
\begin{aligned}
& \hat{\theta}_{\mu \nu}=\lambda_{\mu}^{c} \cdot \lambda_{\nu}^{c} \sigma_{\mu} \cdot \sigma_{\nu}\left(b+d \boldsymbol{\tau}_{\mu} \cdot \boldsymbol{\tau}_{\nu}\right) \cdot V\left(r_{\mu \nu}\right), \\
& V\left(r_{\mu \nu}\right)=V_{0}+K r_{\mu_{\nu}}^{2},
\end{aligned}
$$

其中, $\lambda^{c}$ 是 $S U_{3}^{(c)}$ Gell-Mann 算符, $\boldsymbol{\sigma}$ 是自旋算符, $\boldsymbol{\tau}$ 是味算符, $b 、 d$ 是标志层子相互作用和自 
旋、味关系的常数, $V_{0} 、 K$ 是谐振子势 ${ }^{[4]}$ 的常数. 我们称 (1) 式给出的位 $V_{F}(\boldsymbol{R})$ 为层子集团 折叠位.

对于 $n-p$ 系统, 除了混合比 $b / d$ 作为可调参数外, 其余参数由单核子性质确定如下. 势阱 参数 $b_{0}$ 由核子的方均根半径 $r_{N}^{2}=\frac{3}{2} b_{0}^{2} \sim 0.9 \mathrm{fm}$ 确定, 得 $b_{0}=0.75 \mathrm{fm}$. 假定 $u 、 d$ 层子的质量 相同 $\left(m_{u}=m_{d}=m\right)$, 并由核子磁矩推得 $m=336 \mathrm{MeV}$, 它给出核子结合能为

$$
E_{N}=\langle\Psi(123)|H(123)| \Psi(123)\rangle=M-3 m=-69 \mathrm{MeV},
$$

式中取核子质量 $M=939 \mathrm{MeV}$. 对于谐振子参数, 先用势阱参数 $b_{0}$ 与参数 $K$ 的关系

$$
K=\frac{\hbar^{2}}{16 m(b-5 d) b_{0}^{4}}
$$

定出 $K$ 值，再利用 (7) 式定出 $V_{0}$ 。

图 1 中给出 $E_{n}=0.1 \sim 10^{3} \mathrm{MeV}$ 的 $n-p$ 弹性散射截面, 这是采用 $b / d=-0.38$ 计算的结 果. 从图中看出, 理论值在 $E_{n} \leqslant 30 \mathrm{MeV}$ 内与实验很好地符合; 高能端理论值稍为偏高, 这主 要是没有考虑 $b / d$ 随 $E_{n}$ 变化的结果. 若假定可调参数 $b / d$ 是与能量有关的, 如在高能端取 $b / d=-0.43$, 则可给出较好地符合实验的结果(图 1 中以虚线表示之). 这一问题的唯象核 力分析, 则必须采用 22 个与能量有关的可调参数去符合 ${ }^{[5]}$. 此外, 我们用这些参数 $(b / d=$ - 0.38) 计算了 $n-p$ 结构态, 得到当 $S=1 、 T=0$ 时気核态有最低的能量. 初步结果表明, 采用层子集团折叠位形式, 能从具有囚禁特性的层子相互作用势自然地给出核力. 但这仅仅 是初步的尝试,还需要做更多方面的计算和探索,去进一步检验这一设想的合理性.

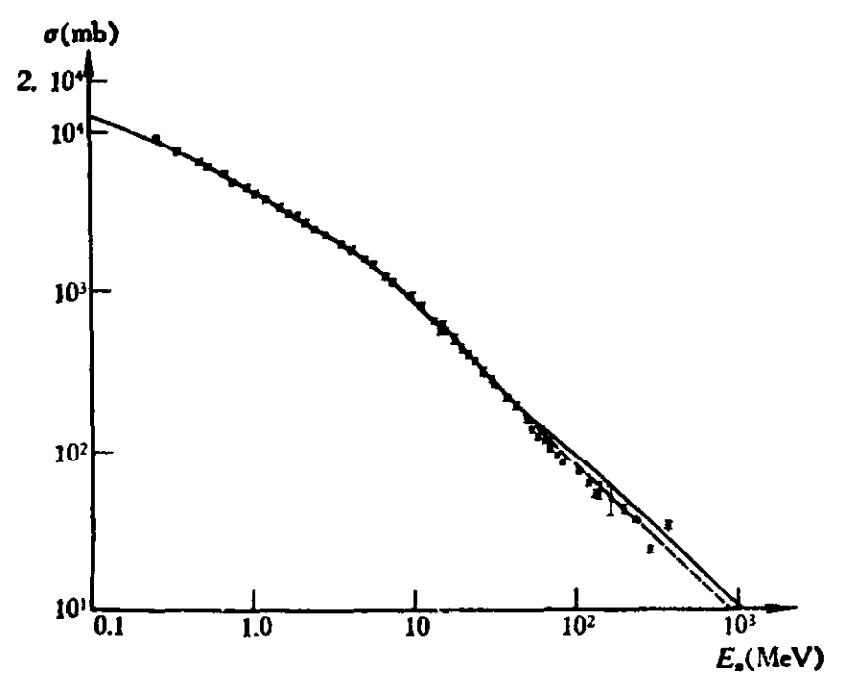

图 1

$-b / d=-0.38 ;---b / d=-0.43$

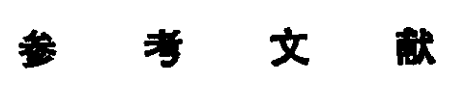

[1] 郑玉明等, 高能物理与核物理, 4(1980), 109 .

[ 2]和 音等,原子核物理(特发表).

[3]卢兆启等, 中国科学, 1979, 771 .

[4] Novikov, V. A. et al., Phys. Reports, 41e (1978), 1.

[5] MacGregor, M. H. et al., Phys. Rev., 173 (1968), 1272.

Arndt, R. A. et al., Phys. Rev., c15 (1977), 1002. 